\title{
El espacio como lugar para la educación cívica: diseño de un patio escolar mediante un proyecto de Aprendizaje - Servicio
}

\author{
(Space as a Place for Civic Education: Design of \\ a School Playground through a Service-Learning \\ Project)
}

\author{
Juan Luis Fuentes \\ Prado Martín-Ondarza \\ Paloma Redondo Corcobado \\ Universidad Complutense de Madrid, UCM (España)
}

DOI: http://dx.doi.org/10.5944/ried.23.1.24496

\section{Cómo referenciar este artículo:}

Fuentes, J. L., Martín-Ondarza, P., y Redondo Corcobado, P. (2020). El espacio como lugar para la educación cívica: diseño de un patio escolar mediante un proyecto de Aprendizaje - Servicio. RIED. Revista Iberoamericana de Educación a Distancia, 23(1), pp. 149-167. doi: http://dx.doi.org/10.5944/ried.23.1.24496

\section{Resumen}

Este artículo presenta los resultados de un proyecto de Aprendizaje-Servicio en el que los espacios físico y virtual desempeñan un papel central e interrelacionado. El proyecto fue desarrollado conjuntamente entre la Universidad Complutense y un centro educativo alejados geográficamente, con el objetivo de transformar pedagógicamente el patio escolar del propio centro. Los estudiantes del Grado en Educación Primaria trabajaron conjuntamente con los alumnos del colegio para el reacondicionamiento del espacio, atendiendo a criterios pedagógicos fundamentados en los contenidos de la asignatura Theory of Education, como la participación ciudadana y comunitaria en el proceso educativo, el compromiso docente, la formación ético-cívica, la inclusión, el cuidado del medio ambiente, el juego al aire libre, entre otros. La metodología utilizada ha sido de carácter cualitativo mediante el análisis de contenido de diferentes documentos, poniendo especial atención en el papel que la tecnología ha desempeñado tanto en la comunicación entre los dos centros, como en la visibilización y reivindicación de una necesidad educativa. Los resultados muestran la reflexión crítica promovida en los participantes por su colaboración en el proyecto, una importante implicación y compromiso cívico con necesidades socioeducativas, la comprensión del papel de las tecnologías en el ejercicio de la ciudadanía contemporánea y en el desarrollo de proyectos de 
Aprendizaje-Servicio, el impacto en la concepción de la identidad docente y el refuerzo de los vínculos entre la universidad, la escuela y la comunidad.

Palabras clave: educación cívica; tecnologías de la información y de la comunicación; relación escuela-comunidad; participación; patio de recreo; distribución del espacio; aprendizajeservicio.

\begin{abstract}
This article presents the results of a Service-Learning project in which physical and virtual spaces have a central and interrelated role. The project was developed by the Universidad Complutense and a geographically distant school with the objective of transforming a playground, from a pedagogical perspective. Future teachers worked together with pupils from the school for the reconditioning of that space, taking into account the contents of the course Theory of Education, such as citizenship and communitarian participation in education, teacher commitment, ethical and civic education, inclusion, environmental education, outdoor games, among others. Method used was qualitative, through content analysis of different documents, paying special attention to the role that technology plays in the process of communication between these two institutions and in the visibility and vindication of an educational need. Results show the critical reflection of participants because of their collaboration in the project, a relevant personal and civic commitment with socio-educational needs, the comprehension of the role of technologies in the contemporaneous citizenship and in Service-Learning projects, the impact in the consideration of teacher identity and the reinforcement of connections between university, school and community.
\end{abstract}

Keywords: civics; information and communication technologies; school-community relationship; participation; playground; space distribution; service-learning.

Tiempo y espacio son dos de los elementos constitutivos de la existencia humana, sin los cuales no es posible comprender lo que somos y lo que hacemos. A lo largo de la historia, no son pocos los filósofos que han considerado su transcendencia en el transcurrir de nuestras vidas. Platón, san Agustín o Heidegger plantearon la compleja y a veces angustiosa relación del ser humano con su irreductible temporalidad, mientras que, en cuanto al espacio, pocos autores han pensado su sentido filosófico como el alemán Otto Friedrich Bollnow (1969), quien concebía los lugares abiertos como necesarios para la apertura del entendimiento y del alma humana.

En el ámbito pedagógico, figuras tan relevantes como Montessori, Fröbel o Steiner consideraban el espacio como un aspecto educativo fundamentaly, lejos de ser entendidos como condicionantes inalterables, proponían profundas adecuaciones del contexto físico de acuerdo con su concepción antropológica. En efecto, las ideas de la escuela Montessori herederas de Rousseau, en las que la autonomía y el crecimiento libre del individuo constituyen algunos de los ejes más significativos sobre los que se articula su modelo educativo, son coherentes con el espacio escolar en el que se 
desarrolla su acción (Montessori, 2014). Muebles pequeños - accesibles a la altura de los infantes-, movibles y escasos - que posibiliten el libre movimiento-, con juegos y materiales didácticos variados y autoevaluables - que facilitan la autonomía y el autocontrol-, así como jardines y parques abiertos - para la interacción con la naturaleza y el ejercicio y juego al aire libre-: son elementos distintivos de muchos centros cuyos proyectos educativos se fundamentan hoy en estas teorías, en las que la arquitectura educativa ha adquirido gran relevancia.

El renacimiento que parecen estar viviendo hoy diversas metodologías activas (Igelmo y Quiroga, 2018; Conde, García Prieto y Delgado-García, 2019) ha puesto también la atención en el espacio físico escolar como condicionante de la acción educativa. Los lugares abiertos son el principal referente de las Bosqueescuelas (Bruchner, 2017), que promueven una educación completamente al aire libre, lo que ya fue señalado por Fröbel, para quien la naturaleza es el espacio en el que el niño encuentra todos los estímulos necesarios para su formación y donde puede desarrollar el juego, en el que confluyen la espontaneidad, la creatividad y la interacción con los otros (Ramos, 2016). Para Steiner, la configuración del espacio, desde el color de las paredes hasta el orden de los materiales, aporta armonía y serenidad en la educación, que en el primer septenio de edad encuentra en el movimiento, la fantasía y el contacto con la naturaleza sus principales notas características (García, 2017). Mientras que, para Malaguzzi, (2011) la importancia educativa que posee el espacio lleva a denominarle en sus escuelas Reggio Emilia como el tercer educador (Beresaluce, 2009).

Investigaciones recientes muestran que el espacio no es una cuestión educativa de segundo orden, sino un condicionante básico que transmite valores (Romañá, 2004); predispone para la adopción de determinados roles de género (Saldaña, Goula, Cardona y Amat, 2018; Subirats y Tomé, 2010); motiva o desmotiva la interacción y la socialización entre alumnos (Czalczynska-Podolska, 2014), y entre alumnos y educadores (Waters y Mainard, 2010); posibilita o imposibilita la expresión corporal, el ejercicio físico y el desarrollo psicomotor (Barbour, 1999); y fomenta el cuidado del medio ambiente y el respeto de la diversidad sociocultural (Uceira, 2009). Además, la implicación de los propios alumnos en la toma de decisiones sobre la configuración de su entorno escolar, como proponía Dewey (1915), constituye aún hoy uno de los retos más importantes en este ámbito (Chancellor y Cevher-Kalburan, 2014), lo que contribuye a evitar diseños estandarizados que resultan poco eficaces en comparación con propuestas del espacio contextualizadas y adaptadas a las necesidades de cada entorno (Jongeneel, Withagen y Zaal, 2015). A pesar de ello, en no pocos casos el potencial pedagógico de los patios escolares es ignorado por administraciones educativas e incluso por los propios educadores (Erdem, 2018).

Paralelamente, en las últimas décadas hemos presenciado la aparición de un nuevo espacio en el que, de manera vertiginosa, hemos comenzado a desarrollar gran parte de las actividades cotidianas, desde las relaciones laborales y personales, hasta las educativas y sociales (Ibáñez-Martín, 2017; Fuentes, Esteban y Caro, 2015). 
La tecnología moderna ha calado tan hondo en nuestras vidas que hemos tenido la necesidad de acuñar nuevos conceptos para describir nuestra presencia en el espacio virtual. Hoy hablamos de ciberespacio y de ciudadanía digital, dos nociones que definen nuevas formas de habitar el espacio virtual (Burbules, 2006), modulando de manera singular la participación ciudadana y los movimientos sociales (Castells, 2012; Jover, González Martín y Fuentes, 2015).

Teniendo en cuenta este contexto, surge la necesidad de reconsiderar la formación de los futuros educadores en cuanto que las tecnologías actuales no pueden ser vistas únicamente como medios e instrumentos, pues mantienen una intensa relación con los fines de la educación (Burbules, 2012) y resultan cada vez más esenciales para el ejercicio de ciertas responsabilidades humanas como la propia ciudadanía (Unesco, 2015).

Este artículo tiene como objetivo presentar los resultados del proyecto Mi recreo, basado en la metodología del Aprendizaje-Servicio y desarrollado conjuntamente entre una universidad y un centro educativo, en el que el espacio físico y virtual desempeñan un papel central. Dicha colaboración tiene como fin el diseño pedagógico de un patio escolar, en el que los estudiantes universitarios y los alumnos del colegio realizan diferentes aprendizajes al mismo tiempo que prestan un servicio a otros. Más concretamente, en este estudio se recogen los resultados de la evaluación cualitativa realizada con los estudiantes de la Universidad Complutense, los alumnos del centro educativo y el equipo directivo.

Entre las características más notables del proyecto se encuentra su doble Aprendizaje-Servicio, donde tanto los estudiantes de la universidad como los alumnos del centro educativo han sido los principales agentes. Asimismo, el proyecto se caracteriza por facilitar la convergencia de principios teóricos que fundamentan el método Montessori y otras propuestas de la Escuela Nueva, donde la infancia es concebida como una etapa vital con singularidad y valor propio (Sanchidrián, 2014), con la metodología del Aprendizaje-Servicio, en la que los estudiantes adquieren un papel protagonista y activo en la modificación de su entorno, evitando actitudes paternalistas y promoviendo el desarrollo de procedimientos democráticos en las instituciones escolares (Puig, Gijón, Martín y Rubio, 2011; Mayor y Rodríguez, 2017). Además, este proyecto tiene la singularidad de que la distancia existente entre ambos centros ha sido superada mediante el uso de la tecnología digital, que ha facilitado la comunicación entre ambas instituciones al mismo tiempo que ha supuesto un factor clave del éxito, especialmente en la difusión y reivindicación de una necesidad ciudadana.

\section{Aprendizaje-Servicio en Theory of Education}

El proyecto Mi recreo encuentra su origen en una demanda realizada por un centro educativo a la universidad. El Colegio de Educación Infantil y Primaria (CEIP) Nuestra Señora de la Soledad es un centro público bilingüe ubicado en Cubas de la 
Sagra, un municipio de la Comunidad de Madrid de unos 6000 habitantes, situado a 42 kilómetros de la Facultad de Educación de la Universidad Complutense.

Tras las obras de ampliación del centro en las que se construyó un nuevo edificio de aulas, el patio escolar no fue modificado. Dos pistas deportivas de cemento constituían el principal espacio para el tiempo de recreo, donde los niños de más edad -niños exclusivamente en masculino- jugaban habitualmente al fútbol, impidiendo más o menos explícitamente a niñas de su misma edad y al resto del alumnado de cursos inferiores disponer de un espacio abierto para el juego y las actividades al aire libre, produciendo así una exclusión y asignación de roles de género a gran parte de los niños del centro. Esta problemática parece ser cada vez más habitual en los centros educativos, lo que está motivando diferentes iniciativas de intervención sobre el tiempo de recreo o el espacio exterior escolar, que posibiliten una mayor inclusión desde la participación comunitaria (Vázquez, Liesa y Lozano, 2017; Miranda, Larrea, Muela, Martínez de Lagos y Barandiaran, 2015).

Sin embargo, en la parte trasera del nuevo edificio del CEIP Nuestra Señora de la Soledad había disponible otro espacio. Una zona de campo irregular que formaba parte del recinto del centro educativo en la que únicamente había tierra y hierbajos, pero que para el equipo directivo del centro guardaba una posible solución para el problema planteado por un recreo únicamente futbolístico y excluyente. De esta manera, surge el proyecto Mi recreo, que tiene como propósito atender a una demanda de reconfigurar un terreno en barbecho para convertirlo en una alternativa lúdico-pedagógica para el alumnado del centro.

Desde la Universidad Complutense se plantea durante el curso 2017-2018 un proyecto de Aprendizaje-Servicio en el que participen los estudiantes del Grupo Bilingüe del Grado en Educación Primaria y, más concretamente, de la asignatura Theory of Education. Esta asignatura, impartida en su totalidad en lengua inglesa, tiene entre sus objetivos fundamentales que los estudiantes conozcan los conceptos y elementos básicos que configuran el quehacer educativo, de acuerdo con los requerimientos de la dignidad humana; analizar los fines y valores de la educación en la sociedad contemporánea; y comprender las condiciones, contextos y agentes de la educación, reflexionando sobre ellos y colaborando en procesos de innovación. Sus contenidos abarcan 6 créditos ECTS e incluyen aspectos como los que se describen en la tabla 1: 
Tabla 1. Dimensión de Aprendizaje del proyecto Mi recreo

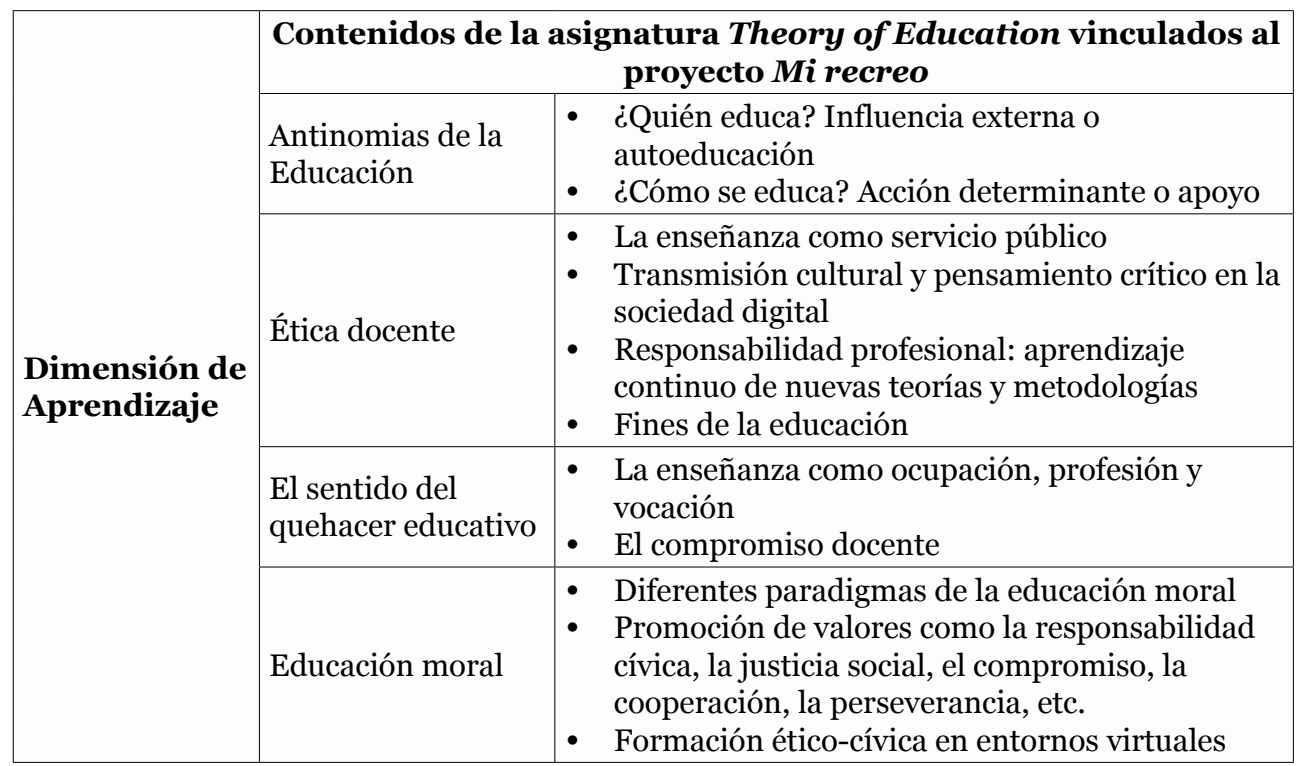

Fuente: Elaboración propia.

Las concepciones pedagógicas de autores como Platón, Aristóteles, Rousseau, Montessori, Froebel, Freire, Bollnow, Dewey, Kohlberg, Noddings y Carr, por citar solo algunos, son estudiadas en esta asignatura. La participación en el proyecto $\mathrm{Mi}$ recreo se propuso como actividad obligatoria evaluable con el objetivo de aplicar en un caso real algunos de los contenidos estudiados y apoyar el desarrollo de competencias específicas y transversales del Grado en Educación Primaria. Fundamentalmente, se trabajaron dos tipos de competencias. Por un lado, aquellas relacionadas con los contenidos disciplinares y la práctica de la lengua inglesa en un contexto académico y profesional. Por otro lado, las competencias referidas al compromiso cívico de los estudiantes universitarios, la vinculación entre teoría y práctica, el liderazgo, el trabajo en equipo, la resolución de problemas, la toma de decisiones y la investigación en el contexto.

Por su parte, el servicio realizado al centro educativo consistió en el diseño de un proyecto educativo para el patio escolar de acuerdo con una serie de criterios derivados del marco teórico de la asignatura:

- La participación del alumnado del centro y de las familias en el diseño y modificación del patio escolar.

- La práctica de valores democráticos en espacios físicos y virtuales.

- $\quad$ El juego y el ocio al aire libre como recurso educativo. 
- La dimensión pedagógica del arte y la cultura.

- La inclusión educativa.

- El cuidado del medio ambiente.

- El compromiso del docente en la acción educativa.

Las principales actividades realizadas en el aula universitaria se organizaron en seis comisiones de trabajo, tal y como se describen en la tabla 2:

Tabla 2. Dimensión de Servicio del proyecto Mi recreo

\begin{tabular}{|c|c|c|}
\hline \multirow{7}{*}{$\begin{array}{l}\text { Dimensión de } \\
\text { Servicio }\end{array}$} & \multicolumn{2}{|c|}{ Comisiones de trabajo } \\
\hline & $\begin{array}{l}\text { 1. Coordinación } \\
\text { general }\end{array}$ & $\begin{array}{l}\text { - Contacto con el centro educativo } \\
\text { - Coherencia de las actividades de cada comisión }\end{array}$ \\
\hline & $\begin{array}{l}\text { 2. Descripción del } \\
\text { contexto }\end{array}$ & $\begin{array}{l}\text { - Estudio de los recursos y necesidades del centro } \\
\text { y de la zona }\end{array}$ \\
\hline & 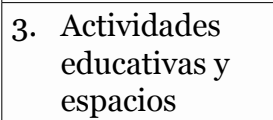 & $\begin{array}{l}\text { - Estudio bibliográfico de experiencias similares } \\
\text { - Elaboración de propuestas }\end{array}$ \\
\hline & $\begin{array}{l}\text { 4. Apoyo } \\
\text { financiero }\end{array}$ & $\begin{array}{l}\text { - } \quad \text { Elaboración de presupuesto } \\
\text { - } \text { de finsulta de convocatoriación } \\
\text { - Estrategias de recaudación de fondos }\end{array}$ \\
\hline & $\begin{array}{l}\text { 5. } \begin{array}{l}\text { Estrategia de } \\
\text { difusión }\end{array}\end{array}$ & $\begin{array}{l}\text { - } \text { Creación de nombre y logo del proyecto } \\
\text { - } \quad \text { Perfiles en RRSS y publicación de noticias } \\
\text { - }\end{array}$ \\
\hline & $\begin{array}{l}\text { 6. Creación de un } \\
\text { vídeo }\end{array}$ & $\begin{array}{l}\text { - Presentación del proyecto y del trabajo de las } \\
\text { comisiones }\end{array}$ \\
\hline
\end{tabular}

Fuente: Elaboración propia.

Desde el centro educativo se creó el Consejo de los Niños del Patio, un órgano de representación estudiantil formado por alumnos de educación primaria que tenía la responsabilidad de organizar las actividades del alumnado del centro en torno a la reconversión del patio escolar. Asimismo, era la referencia para el contacto con la universidad, las familias, la comunidad educativa en general y otras instituciones públicas. Además, las profesoras del colegio proporcionaron a sus alumnos una plantilla del área del patio con el objetivo de que dibujaran y describieran las actividades que querrían realizar en el espacio. Estos materiales fueron objeto de estudio tanto del Consejo de los Niños del Patio como del grupo de estudiantes universitarios para la elaboración del diseño final. 


\section{Metodología}

La metodología utilizada en este estudio es de corte cualitativo y evaluativo. Los participantes que componen la muestra utilizada han sido: 22 estudiantes de la asignatura Theory of Education del Grado de Educación Primaria; 18 estudiantes del Consejo de los Niños del Patio, de $1^{\circ}$ a $6^{\circ}$ de Educación Primaria, y 2 componentes del equipo directivo (directora y jefa de estudios) del CEIP Ntra. Sra. de la Soledad.

La información analizada ha sido extraída de 57 documentos de diferente naturaleza:

- Memorias de reflexión elaboradas por los estudiantes sobre su participación y valoración del proyecto ApS, de las cuales: 22 corresponden a estudiantes de la asignatura Theory of Education; 13 al grupo de estudiantes que continuaron voluntariamente en el proyecto el cuatrimestre posterior a haber finalizado esta asignatura y 18 a alumnos que conformaban el Consejo de los Niños del Patio. En las memorias de los estudiantes universitarios se propuso explícitamente reflexionar sobre el papel que la tecnología había desempeñado en el desarrollo del proyecto.

- Entrevista semiestructurada realizada de manera conjunta a la directora y la jefa de estudios del CEIP, con el fin de recabar su valoración del desarrollo del proyecto y la colaboración entre escuela y universidad.

- Transcripciones de los documentos audiovisuales correspondientes a las intervenciones de representantes de los estudiantes del proyecto, tanto de la universidad como del Consejo de los Niños del Patio, en 2 mesas redondas realizadas en la Semana Cultural de la Facultad de Educación de la Universidad Complutense, con el objetivo de presentar el proyecto.

- Transcripción de la grabación del Grupo de Discusión realizado con 13 estudiantes que permanecieron en el proyecto hasta el final del curso 2017-2018. Las líneas principales de discusión que se propusieron en relación a su participación en este proyecto fueron: adquisición de aprendizajes curriculares; desarrollo social y personal; detección de carencias formativas; implementación de proyectos ApS en los estudios de grado universitarios; impacto social del servicio que realizaron y percepción de la institución donde habían desarrollado el servicio.

Con la información obtenida se ha realizado un análisis del contenido en función de las principales dimensiones de la metodología del Aprendizaje-Servicio, así como de otras que han resultado ser relevantes para el proyecto y sus participantes. La utilización del software Atlas.ti 8 ha permitido codificar la información de acuerdo a las categorías y subcategorías contenidas en la tabla 3. Los resultados finales han sido sometidos a un proceso de triangulación en el que participó el equipo de investigadores responsables del estudio. 
Tabla 3. Categorías y subcategorías del análisis

\begin{tabular}{|c|c|}
\hline Categorías & Subcategorias \\
\hline \multirow{2}{*}{ Aprendizaje } & Dimensión Personal \\
\cline { 2 - 2 } & Dimensión Social \\
\cline { 2 - 2 } Servicio & Dimensión Curricular \\
\hline \multirow{2}{*}{$\begin{array}{c}\text { Aportación de la tecnología al desarrollo del } \\
\text { proyecto }\end{array}$} & Sentido \\
\cline { 2 - 2 } & Impacto \\
\hline
\end{tabular}

Fuente: Elaboración propia.

\section{Resultados}

Aprendizaje

\section{a. Dimensión personal}

La participación de los estudiantes en el proyecto muestra un alto desarrollo intrapersonal que tiene como motor la reflexión crítica. Los alumnos reflejan haber desarrollado su capacidad para problematizar la realidad y buscar soluciones a necesidades sociales de forma creativa, aprendiendo con una alta motivación y con gran iniciativa hacia las innovaciones.

Además, el proyecto les ha permitido tomar conciencia y adquirir conocimiento sobre las realidades que les rodean, físicas y virtuales, en las que van a realizar su actividad profesional. Dicho conocimiento les ha ayudado, según explica una estudiante universitaria, a "encontrar un equilibrio entre los límites temporales, sociales, espaciales y económicos” que indudablemente cualquier proyecto posee y a aprender de los errores cometidos.

Por otro lado, resulta llamativa la alusión común a la vocación tanto de los estudiantes universitarios como de los alumnos del colegio, en dos sentidos distintos. Los primeros parecen confirmar mayoritariamente la decisión de haber optado por la educación en su formación universitaria, lo que ha producido, al mismo tiempo, una reflexión contextualizada sobre uno de los contenidos principales de la asignatura Theory of Education, referido a las diferencias entre ocupación, profesión y vocación (tabla 1), siguiendo los planteamientos de Carr (2002). Por su parte, los alumnos del colegio manifiestan que "es posible que les hayan descubierto una posible vocación" y que "de mayor quieren ir a la universidad a estudiar y aprender", lo que pone en evidencia algunos de los beneficios del contacto entre la universidad y los centros educativos, como la apertura a nuevos horizontes vitales y profesionales, que resulta especialmente significativa en entornos rurales. 


\section{b. Dimensión social}

Gran parte del aprendizaje realizado a través de la participación en el proyecto tiene que ver con el desarrollo interpersonal. Conceptos como "ayuda al otro", "colaboración", "participación de todos", búsqueda de la "implicación social de la comunidad educativa" o "trabajo en red", están muy presentes en los discursos de los participantes, tal y como se observa en los documentos analizados.

Los alumnos verbalizan haber aprendido a trabajar en grupo por un objetivo común, adquiriendo y poniendo en práctica habilidades sociales y comunicativas para la toma de decisiones grupal, así como la capacidad de hablar en público, negociar y consensuar diferentes puntos de vista. Reconocen la importancia que estas capacidades poseen en la cultura democrática y manifiestan un mayor sentimiento de pertenencia al grupo, lo que supone un mayor compromiso con el proyecto y con la comunidad de referencia.

Cabe destacar especialmente las repetidas referencias a valores inclusivos. Los alumnos del colegio manifiestan que necesitan un patio que se adapte a las necesidades de todos, han aprendido que quieren "un patio inclusivo para chicos y chicas" en el que "no se diga este columpio es de chicas o de chicos, sino que no haya diferencia". Por su parte, los alumnos de la universidad reflexionan sobre la importancia de la tecnología en la inclusión social, concretamente en relación a temas como el lenguaje inclusivo en la red o su capacidad para ponernos en contacto con realidades diferentes a las propias, aprendiendo la gran magnitud de la diversidad humana y la oportunidad que la tecnología brinda en este sentido en el ámbito educativo.

De esta manera, "empatía”, "inclusión”, "respeto", “cooperación” y "autogestión" aparecen como principales motores sociales del proyecto, así como grandes aportaciones al desarrollo social de sus participantes que desembocan en una alta implicación social con el medio que les rodea.

\section{c. Dimensión curricular}

Los estudiantes universitarios muestran en los documentos analizados un notable aprendizaje de conceptos curriculares de la asignatura Theory of Education, contextualizados en la situación real de un centro educativo. Esta aplicación práctica de la teoría pedagógica aporta motivación por la formación universitaria al alumnado y favorece la interiorización de los aprendizajes de manera significativa. Manifiestan haber aprendido acerca de la importancia del contexto en el que los niños juegan, de la necesidad educativa de disponer de un espacio donde, mediante la actividad lúdica y no dirigida, desarrollen capacidades cognitivas, sociales y emocionales. Asimismo, son conscientes de que la inclusión no es únicamente un concepto teórico deseable, sino también y especialmente una realidad práctica que requiere ser contemplada de manera transversal en distintos tiempos y espacios de convivencia humana. Para 
ello, los estudiantes destacan que el pensamiento crítico tiene un papel fundamental en el cuestionamiento de las estructuras sociales establecidas y en la comprensión de la realidad social.

Con respecto a la metodología del Aprendizaje-Servicio, afirman haber aprendido que "realmente es una pedagogía que combina el curriculum académico y el servicio a la comunidad", que promueve la responsabilidad social y requiere de un alto compromiso docente para su desarrollo. Asimismo, apuntan algunas limitaciones como su sostenibilidad a lo largo del tiempo por las dificultades organizativas que implican en la comunidad escolar. De igual manera, reconocen el enriquecimiento que supone para su formación como educadores, pero advierten una falta de formación en esta metodología que, en su opinión, debería formar parte de la formación universitaria, no sólo en los Grados vinculados a la educación, sino también en otras disciplinas académicas, por el compromiso cívico que el ApS fomenta.

\section{Servicio}

\section{a. Sentido}

El sentido del servicio para los protagonistas del proyecto se centra fundamentalmente en ofrecer ayuda a otros para promover su bienestar, dando respuesta a una necesidad social de su realidad más próxima, a la vez que se realiza un aprendizaje. Para la mayoría de los estudiantes universitarios, este trabajo ha cobrado sentido a través de la toma de conciencia de que uno de los principales objetivos de su futura profesión docente es el cuidado de la infancia. Son numerosos los comentarios en los que exponen que su motivación y su recompensa al trabajo realizado se ha encontrado en "ver a los niños felices".

Otro punto fundamental es la valoración por su parte de la función social de la educación y la necesidad de los aprendizajes en valores democráticos y ético-cívicos, lo cual se ha canalizado fundamentalmente en la enseñanza-aprendizaje del uso de las tecnologías, dada la importancia que éstas han cobrado a lo largo de todo el proceso.

En cuanto a los alumnos que han formado parte del Consejo de los Niños del Patio, el proyecto ha cobrado sentido gracias al impacto que ha tenido en su desarrollo y concientización social. Ponen el foco especialmente en la adquisición de habilidades sociales, en el trabajo en equipo y en la responsabilidad que los ha llevado a buscar soluciones a una necesidad que consideraban esencial para sus compañeros más pequeños. En las reflexiones sobre su experiencia declaran estar orgullosos de su labor y participación como miembros de este órgano de representación, así como de trabajar para conseguir el patio adecuado para sus compañeros de $1^{\circ}$ y $2^{\circ}$ de primaria. Todo ello puede asociarse a trabajar, aprender y aunar esfuerzos para conseguir el bien común. Por tanto, se observa la importancia que puede tener la implementación de proyectos de Aprendizaje-Servicio en la implicación social de los 
estudiantes y en la generación de una cultura democrática en los centros escolares. Así lo expresa uno de los alumnos de educación primaria:

Me he sentido muy orgulloso de haber sido elegido para pertenecer a este consejo e intentar conseguir algo tan importante como lo es un patio nuevo en el que puedan jugar sin ningún peligro los peques del cole.

b. Impacto

El impacto del servicio puede encontrarse en los siguientes ámbitos:

- Percepción de la colaboración universidad-centro escolar. Este cambio se produce principalmente sobre las expectativas que las personas responsables del proyecto en el colegio tenían inicialmente. El equipo directivo explica que, tras cierta incertidumbre y desconfianza inicial, el encuentro y la colaboración con la Universidad ha sido un hecho fundamental que les ha impulsado y aportado seguridad, apoyo, motivación y respuestas a las necesidades del servicio planteado. Asimismo, ha dado lugar a la reflexión sobre la importancia de la relación universidad-comunidad en la formación de docentes implicados socialmente y coherentes con las demandas actuales de la sociedad. Todo ello pone de relieve la responsabilidad social de las instituciones educativas de educación superior.

- Desarrollo de otros proyectos de Aprendizaje-Servicio. El impacto del desarrollo de este proyecto se observa también en la promoción de otros cinco proyectos de Aprendizaje-Servicio en el centro educativo. Si bien ya habían desarrollado anteriormente otras iniciativas similares sin conocer el Aprendizaje-Servicio, según explican las profesoras del equipo directivo, el proyecto ha favorecido la sistematización de las experiencias y el establecimiento de criterios que contribuyan a una mayor conexión curricular de las actividades de servicio realizadas.

- Implicación de la comunidad educativa. El impacto en la comunidad educativa se evidencia tanto en el análisis de los eventos de presentación del proyecto y en la inauguración del patio, como en la colaboración de las familias en el proceso de acondicionamiento del terreno. Según manifiesta el equipo directivo, el análisis del material audiovisual correspondiente, el gran número de asistentes, la implicación de docentes y estudiantes de las diferentes etapas educativas, de las familias y la importante cantidad de dinero recaudado para las obras y adecuación del patio, queda patente el éxito del servicio y la implicación obtenida en el proyecto. A modo de ejemplo, la satisfacción de las familias quedó reflejada en un tuit de una madre del colegio tras finalizar uno de los eventos: "Esto 
es un proyectazo, bien organizado, pensando en todos y participando todos. Entusiasmada de lo vivido y lo aprendido".

- Impacto sobre personas ajenas al proyecto procedentes de contextos cercanos a los estudiantes de la Facultad de Educación. La difusión de la experiencia y la satisfacción con el resultado produce un efecto "bola de nieve", creando interés por conocer en qué consiste esta metodología docente y por participar en proyectos de Aprendizaje-Servicio. Así lo expresa una de las estudiantes:

Si sabes de alguien que participa y te anima a participar parece que todo se va retroalimentando y luego se lo dices a no sé quién y se va apuntando, y algo que era muy chiquitito se crea una bola muy grande y yo creo que es lo que ha pasado aquí. Ha sido una bola de nieve, ha empezado algo muy pequeñito y al final ha sido una cosa que tiene un montón de ramificaciones.

- Impacto en la construcción de la identidad docente de los alumnos de la Facultad de Educación. La participación en este proyecto con trasfondo social y el contacto directo con la realidad educativa ha llevado a los alumnos a una reflexión sobre lo que implica ser educador y el compromiso ético que supone desarrollar la práctica docente en educación primaria: objetivos, responsabilidades, valores, conocimientos, etc. Por tanto, les sitúa a través de un aprendizaje experiencial y significativo en el papel de su futura profesión, modifica su percepción de la escuela y la formación que han recibido para situarse en el rol de docentes dentro de la complejidad real de un contexto escolar y social.

- Impacto en las calificaciones de los estudiantes y del profesorado universitario. La valoración positiva de los estudiantes sobre el gran alcance de los aprendizajes conseguidos se refrenda en las calificaciones finales de la asignatura Theory of Education, en la que la nota media fue significativamente más alta en comparación con los dos cursos anteriores, mientras que la evaluación cuantitativa del profesorado en el programa Docencia obtuvo también un mejor resultado que en los dos años previos.

\section{Aportación de la tecnología al desarrollo del proyecto}

Como se ha podido observar en algunos de los apartados anteriores, la tecnología ha tenido un papel muy significativo en el desarrollo del proyecto de AprendizajeServicio Mi recreo. Por ello, se recogen en este apartado algunas de las aportaciones más relevantes destacadas por los participantes.

Las herramientas tecnológicas más utilizadas y citadas han sido los motores de búsqueda de Internet, el correo electrónico, el campus virtual, las redes sociales y los programas para la edición de vídeo. Estas herramientas de carácter sencillo en su uso y accesibilidad han permitido, en primer lugar, salvar las distancias entre la universidad y el centro educativo, ampliando así las posibilidades de desarrollo de 
proyectos de Aprendizaje-Servicio y de atención a necesidades sociales, que no se restringen a las del entorno más inmediato. En efecto, los motores de búsqueda son señalados por los estudiantes como los medios que han facilitado una investigación sobre el contexto en el que se encuentra el centro educativo, los recursos de la zona y las características de la población, así como sobre los recursos propios de la universidad para el desarrollo del proyecto.

En segundo lugar, los canales tecnológicos han permitido transmitir mensajes que incluyen no sólo texto escrito, sino también imágenes, movimientos y música, ampliando de esta manera los lenguajes comunicativos. Así lo señala una de las estudiantes universitarias:

Gracias a la tecnología digital hemos podido estar conectados con el colegio desde la Facultad de Educación, nos hemos comunicado con ellos vía e-mail, además de que también podían ver nuestras publicaciones en redes sociales. También hicimos un video que nos ayudó bastante a que se viera nuestro progreso y nuestras ganas. Por lo que considero que hemos estado bastante unidos al colegio gracias a la tecnología digital.

En tercer lugar, los estudiantes universitarios y las profesoras del equipo directivo señalan que el trabajo de difusión tecnológica ha sido un punto clave en el éxito del proyecto, que ha implicado diferentes estrategias como la creación de perfiles en redes sociales (Facebook y Twitter) y en plataformas destinadas a la recaudación de financiación (Change.org), la elaboración del vídeo en el que presentaban el proyecto, el proceso del trabajo realizado y sus propuestas en primera persona. Dos estudiantes así lo expresan:

Utilizar medios visuales como son la grabación de un vídeo pueden resultar muy útiles y tienen una mayor eficacia y alcance para el público.

Las tecnologías digitales han jugado uno de los papeles principales en el desarrollo del proyecto. La creación de las cuentas en las redes sociales ha sido clave para la difusión del proyecto. En ellas se hacían publicaciones en las que se explicaba el proceso de desarrollo del proyecto, se publicaban los futuros eventos que se realizarían tanto fuera como dentro del colegio e información relevante sobre el proyecto. Además, la involucración de los alumnos del centro en la elaboración del proyecto consiguió que se llegase a hablar de ello hasta en la radio, cosa que le dio aún más difusión.

En este sentido, la directora del centro educativo va más allá y apunta que la campaña de difusión ha conseguido la implicación de la comunidad educativa y los apoyos externos necesarios:

Las estudiantes de la Facultad han aportado difusión y gracias a ellas hemos conseguido el apoyo del Ayuntamiento, de las empresas, de los padres de los alumnos y de los propios alumnos. 
En cuarto lugar, la tecnología ha facilitado la creación de espacios virtuales de trabajo en equipo, fundamentalmente en el aula virtual de la universidad, en el que se han compartido recursos bibliográficos, audiovisuales, noticias, avances, que resultan imprescindibles para el trabajo cooperativo en un proyecto conjunto. No obstante, en cuanto que el uso de este espacio está limitado a usuarios de la universidad, su apertura a otros participantes externos no ha sido posible, lo que hubiera sido deseable para un diálogo más fluido entre universidad y centro educativo. Además, al ser espacios cerrados, ha sido necesaria la creación de otros espacios abiertos alternativos que permitieran la difusión del proyecto de ApS, como las redes sociales.

En quinto lugar, la utilización de la tecnología ha promovido la reflexión sobre su uso en los participantes a diferentes niveles. Se observa una visión crítica sobre las redes sociales, lo que implica no solo una valoración positiva de sus aportaciones, sino también una identificación de sus límites y riesgos. Los estudiantes universitarios reconocen la relevancia que la tecnología posee en el ejercicio de una cultura de servicio global y una ciudadanía digital que ponga el acento en la cooperación entre personas de diferentes partes del mundo. Al mismo tiempo, advierten de los riesgos existentes en su utilización por la dimensión pública de la participación digital en Internet, especialmente en regímenes no democráticos, pero también en sociedades escasamente tolerantes con la diversidad de opiniones. Esto ha provocado también reflexiones sobre su propio comportamiento en la red y los peligros que afrontan en su uso diario, así como los límites en el uso de Internet y redes sociales en menores de edad, debido a su mayor vulnerabilidad frente a los adultos.

Finalmente, resultan también significativas las demandas que los estudiantes universitarios realizan con motivo de su participación en el proyecto sobre sus necesidades de formación docente, que se dirigen a la eficacia y potencialidad de la tecnología y las redes sociales no solo en su dimensión técnica, sino también en la ético-cívica y, más especialmente, a la responsabilidad social que ello implica. La mayoría reconoce haber aprendido sobre la gran utilidad de la tecnología como recurso educativo. Sin embargo, también reflexionan sobre el abandono de las formas "rudimentarias de crear espacios pedagógicos" en beneficio de los recursos tecnológicos, que, según indican, no siempre son más adecuados que los tradicionales.

\section{CONCLUSIONES}

Las aportaciones que el Aprendizaje-Servicio realiza a la formación de los estudiantes son muy variadas (Furco y Root, 2010) y se demuestran tanto en la investigación educativa como en la práctica y la experiencia de cada vez más proyectos desarrollados en diferentes niveles educativos. En este artículo, hemos presentado un estudio de caso con singularidades notables, tanto por la diversidad de los protagonistas, como por la centralidad de los espacios físico y virtual y la relevancia de este último en el desarrollo y consecución del proyecto. Algunas de las 
conclusiones más destacables que se derivan de su análisis y que pueden orientar futuras investigaciones son las siguientes:

- Los estudiantes universitarios demandan mayor formación, tanto en lo que respecta al potencial pedagógico de la tecnología, como en el uso del AprendizajeServicio.

- El Aprendizaje-Servicio constituye una estrategia apropiada para conectar la universidad con los centros educativos y con la sociedad en general (Vázquez, Liesa y Lozano, 2017), posibilita generar la imagen de una universidad que atiende a las necesidades sociales, al mismo tiempo que evita una posición paternalista cuando establece una colaboración interinstitucional en la que todos los individuos aportan en función de sus posibilidades.

- En los grados universitarios de carácter pedagógico, el desarrollo de proyectos de Aprendizaje-Servicio tiene una finalidad añadida a las habituales en la universidad. Junto con la formación en contenidos curriculares, la formación ético-cívica y la prestación de un servicio, cabe añadir el objetivo del aprendizaje metodológico. Es decir, no solo aprenden como en este caso, teoría de la educación de manera significativa y contextualizada, sino que al mismo tiempo conocen y practican una metodología de gran utilidad para su formación como educadores. De esta forma, contribuiremos a la inclusión del Aprendizaje-Servicio en la dinámica cotidiana de los centros.

- Cabe reconsiderar algunas de las dificultades aparentes en el desarrollo de proyectos de Aprendizaje-Servicio: por un lado, su potencial en asignaturas de carácter teórico, lo que se muestra accesible en disciplinas educativas, debido al carácter aplicado que poseen en ellas las cuestiones teóricas y filosóficas (García Amilburu y García Gutiérrez, 2012); por otro lado, la menor experiencia y formación de los estudiantes de primeros cursos universitarios, lo que si bien puede restar capacidad de actuación en el servicio, inserta a los individuos en una realidad motivadora en la que adoptan de manera temprana el papel de educadores y perciben la formación universitaria desde una perspectiva diferente y significativa. Las asignaturas ya no son percibidas como obstáculos a superar y aprobar, sino como contenidos necesarios para su desempeño profesional.

- Finalmente, la tecnología realiza aportaciones significativas a los proyectos de Aprendizaje-Servicio. Junto a las ya señaladas de comunicación entre instituciones que posibilitan superar la distancia entre ellos, y las de visibilización y reivindicación de necesidades sociales, puede añadirse la facilidad de plantear esta actividad de forma obligatoria en los planes de estudio, al no requerir que los estudiantes tengan que desplazarse necesariamente, cambiando su lugar de estudios y ampliando sus horarios. 


\section{NOTAS}

1. Los autores de este artículo agradecemos a los estudiantes del Grupo Bilingüe del Grado en Educación Primaria de la Universidad Complutense, a Ángela González Caro (estudiante del Grado en Educación Social), al alumnado, profesorado, equipo directivo y familias del CEIP Nuestra Señora de la Soledad, al CTIF Madrid-Sur y a sus profesionales, y a todas las instituciones que han colaborado en hacer posible este proyecto.

\section{REFERENCIAS}

Barbour, A. C. (1999). The Impact of Playground Design on the Play Behaviors of Children with Differing Levels of Physical Competence. Early Childhood Research Quarterly, 14(1), 75-98. doi: $\quad$ https://doi.org/10.1016/So8852006(99)80007-6

Beresaluce, R. (2009). Las escuelas reggianas como modelo de calidad en la etapa de educación infantil. Aula abierta, 37(2), 123-130.

Bollnow, O. F. (1969). Hombre y espacio. Barcelona: Labor.

Bruchner, P. (2017). Bosquescuela. Guía para la educación infantil al aire libre. Rodeno: Valencia.

Burbules, N. C. (2006). Rethinking Dialogue in Networked Spaces. Cultural Studies - Critical Methodologies, 6(1), 107-122. doi: https://doi. org/10.1177/1532708605282817

Burbules, N. C. (2012). Ubiquitous Learning and the Future of Teaching. Encounters in Theory and History of Education, 13, 3-14. doi: https://doi.org/10.24908/eoeese-rse.v13io. 4472

Carr, D. (2002). Making Sense of Education: An Introduction to the Philosophy and Theory of Education and Teaching. Hoboken: Routledge.

Castells, M. (2012). Redes de indignación y esperanza. Madrid: Alianza.

Chancellor, B., y Cevher-Kalburan, N. (2014). Comparing and Contrasting Primary School Playgrounds in Turkey and Australia. International Education Journal: Comparative Perspectives, 13(2), 1-19.

Conde, S., García Prieto, F. J., y DelgadoGarcía, M. (2019). Diseño y validación de un instrumento para analizar el trabajo por rincones en las aulas de Educación Infantil. Estudios sobre educación, 36, 53-83. doi: https://doi. org/10.15581/004.36.53-83

Czalczynska-Podolska, M. (2014). The Impact of Playground Spatial Features on Children's Play and Activity Forms: An Evaluation of Contemporary Playgrounds' Play and Social Value. Journal of Environmental Psychology, 38, 132142. doi: https://doi.org/10.1016/j. jenvp.2014.01.006

Dewey, J. (1915). The Schools of Tomorrow. New York: Dutton \& Company.

Erdem, D. (2018). Kindergarten Teachers' Views About Outdoor Activities. Journal of Education and Learning, 7(3), 203217. doi: https://doi.org/10.5539/jel. v7n3p159

Fuentes, J. L., Esteban, F., y Caro, C. (2015). Vivir en Internet. Madrid: Síntesis.

Furco, A., y Root, S. (2010). Research Demonstrates the Value of Service Learning. Phi Delta Kappan, 91(5), 16-20. doi: https://doi. org/10.1177/003172171009100504

García Amilburu, M., y García Gutiérrez, J. (2012). Filosofía de la Educación. 
Cuestiones de hoy y de siempre. Madrid: Narcea.

García, A. (2017). Otra educación es posible. Albuixech: Litera Libros.

Ibáñez-Martín, J. A. (2017). Horizontes para los educadores. Madrid: Dykinson.

Igelmo-Zaldívar, J., y Quiroga Uceda, P. (2018). La pedagogía ligera en tiempos hipermodernos: el homeschooling, las escuelas Waldorf y la nueva pedagogía ignaciana. Teoría de la educación. Revista Interuniversitaria, 30(1), 7594. doi: http://dx.doi.org/10.14201/ teoredu3017594

Jongeneel, D., Withagen, R., y Zaal, F. (2015). Do Children Create Standardized Playgrounds? A Study on the GapCrossing Affordances of Jumping Stones. Journal of Environmental Psychology, 44, 45-52. doi: https://doi.org/10.1016/j. jenvp.2015.09.003

Malaguzzi, L. (2011). La educación infantil en Reggio Emilia. Barcelona: Octaedro.

Mayor, D., y Rodríguez, D. (2017). Aprendizaje-Servicio: una práctica pedagógica que promueve la participación del estudiantado para la mejora escolar y social. Revista Complutense de Educación, 28(2), 555-571. doi: https:// doi.org/10.5209/rev RCED.2017.v28. n2.49623

Miranda, N., Larrea, I., Muela, A. Martínez de Lagos, A., y Barandiaran, A. (2015). Mejora del espacio exterior escolar desde la participación comunitaria. Participación educativa, 4(7), 160-167.

Montessori, M. (2014). El método de la pedagogía científica: aplicado a la educación de la infancia. Madrid: Biblioteca Nueva.

Puig, J. M., Gijón, M., Martín, X., y Rubio. L. (2011). Aprendizaje-Servicio y Educación para la Ciudadanía. Revista de Educación, $\mathrm{n}^{0}$ extraordinario, 45-67.

Ramos, S. (2016). Un método educativo para la infancia: el de Fröbel. En C. Sanchidrián y J. Ruiz Berrio (Coords.), Historia y perspectiva actual de la educación infantil (113-132). Barcelona: Graó.

Romañá, T. (2004). Arquitectura y educación: perspectivas y dimensiones. Revista Española de Pedagogía, 67 (228), 199-220.

Saldaña, D., Goula, J., Cardona, H., y Amat, C. (2018). El pati de l'escola en igualtat. Guia de diagnosi $i$ d'intervenció amb perspectiva de gènere. Barcelona: Equal Saree.

Sanchidrián, C. (2014). Introducción. En M. Montessori, El método de la Pedagogía científica. Madrid: Biblioteca Nueva.

Subirats, M., y Tomé, A. (2010). Balones fuera: reconstruir los espacios desde la coeducación. Madrid: Octaedro.

Uceira, E. (2009). El patio escolar como espacio de integración social. Quaderns digitals: Revista de Nuevas Tecnologías y Sociedad, 6o, 1-8.

Unesco (2015). Replantear la educación: ¿Hacia un bien común mundial? París: UNESCO.

Vázquez, S., Liesa, M., y Lozano, A. (2017). Recreos Cooperativos e Inclusivos a través de la metodología de Aprendizaje-Servicio. Revista Electrónica Interuniversitaria de Formación del Profesorado, 2o(1), 173-185. doi: http://dx.doi.org/10.6018/ reifop.20.1.213181

Waters, J., y Maynard, T. (2010). What's So Interesting Outside? A Study of Child Initiated Interaction With Teachers in the Natural Outdoor Environment. European Early Childhood Education Research Journal, 18(4), 473-483. doi: https://doi. org/10.1080/1350293X.2010.525939 


\section{PERFIL ACADÉMICO Y PROFESIONAL DE LOS AUTORES}

Juan Luis Fuentes. Profesor Ayudante, Doctor del Departamento Estudios Educativos de la Facultad de Educación, Universidad Complutense de Madrid, donde es miembro del Grupo de Investigación "Cultura Cívica y Políticas Educativas" y Delegado del Decano para Relaciones Comunitarias. Sus líneas de investigación se sitúan en torno a la teoría y la filosofía de la educación, la educación del carácter y la utilización de las TIC en el ámbito educativo desde una perspectiva ético-cívica.

E-mail: jlfuente@ucm.es

Prado Martín-Ondarza. Personal Investigador en Formación (Programa FPU del Ministerio de Ciencia, Innovación y Universidades) adscrita al Departamento de Estudios Educativos de la Facultad de Educación, Universidad Complutense de Madrid, donde es miembro del Grupo de Investigación "Cultura Cívica y Políticas Educativas". Sus líneas de investigación se sitúan en torno a la etapa de Educación Infantil, concretamente relacionadas con el juego, la diversidad y la cultura inclusiva en contextos educativos formales, así como en metodologías docentes que pueden ayudar a generarla (Grupos Interactivos y Aprendizaje-Servicio).

E-mail: mmartino@ucm.es

Paloma Redondo Corcobado. Doctoranda en el Programa de Doctorado en Educación en la Facultad de Educación de la Universidad Complutense de Madrid, participante en el Grupo de Investigación "Cultura Cívica y Políticas Educativas" adscrito al Departamento de Estudios Educativos. Sus trabajos de investigación están relacionados con la educación ético-cívica, y más específicamente en torno a la metodología Aprendizaje-Servicio.

E-mail:paloredo@ucm.es

Dirección:

Facultad de Educación - Centro de Formación del Profesorado

Universidad Complutense de Madrid

Rector Royo Villanova, $\mathrm{n}^{0} 128040$

Madrid (España)

Fecha de recepción del artículo: 23/05/2019

Fecha de aceptación del artículo: 22/06/2019

Fecha de aprobación para maquetación: 11/08/2019 